\title{
Alloying in Flexible Transition-metal Chalcogenide Nanowires
}

\author{
Junhao Lin ${ }^{1,2,3}$, Yuyang Zhang ${ }^{2}$, Wu Zhou ${ }^{3}$, Sokrates T. Pantelides ${ }^{2,3}$ \\ 1. National Institute of Advanced Industrial Science and Technology (AIST), Tsukuba 305-8565, Japan \\ 2. Department of Physics and Astronomy, Vanderbilt University, Nashville, TN 37235, USA \\ 3. Materials Science \& Technology Division, Oak Ridge National Laboratory, Oak Ridge, TN 37831, \\ USA
}

With the advent of research on transition-metal dichalcogenide monolayers (TMDC) as a promising monolayer semiconductor for applications in flexible nanoelectronics, transition-metal chalcogenide (TMC) nanowires become one of the important building blocks due to their intrinsic 1D nature and metallicity [1]. Early studies report direct synthesis of TMC bundled nanowires in the form of Mo6 $\mathrm{X}_{6}$ and $\mathrm{Mo}_{6} \mathrm{~S}_{9-\mathrm{x}} \mathrm{I}_{\mathrm{x}}(\mathrm{X}=\mathrm{S}$ or $\mathrm{Se})$ on various substrates [2-5]. Fabrication of free-standing and isolated individual TMC nanowire within a TMDC 2D material matrix was recently achieved by electron-beam irradiation of TMDC monolayers [1,6,7]. However, up till now, there are few reports on the tuning possibility of the electronic properties in individual TMC nanowires, presumably due to the difficulties in manipulating the atomic structure and chemical composition of individual nanowires during the fabrication.

Here we show that $\mathrm{MoS}_{1-\mathrm{x}} \mathrm{Se}_{\mathrm{x}}$ and $\mathrm{Mo}_{1-\mathrm{x}} \mathrm{W}_{\mathrm{x}} \mathrm{S}$ alloying nanowires can be controllably fabricated by electron beam sculpting technique [1] in a scanning transmission electron microscope (STEM) [8]. The experiments were performed at ORNL on an aberration-corrected Nion UltraSTEM-100 operated at 60 and $100 \mathrm{kV}$. We successfully fabricated these nanowires from their parent alloyed monolayers and vertically stacked bilayer heterostructure, both of which serve as the reservoir of S and Se (or Mo and W) atoms during the fabrication of the alloyed nanowires. By combining the atom-by-atom intensity quantification analysis of the STEM images with density functional theory (DFT), we directly map out their atomic structure and chemical stoichiometry (Figure 1), which enable us to explore the possibility of tuning the electronic properties in these alloyed nanowires.

We first illustrate that both Se and $\mathrm{W}$ substitutions in MoS nanowires do not have preferential substitutional sites, as demonstrated by the random distribution of the substitutional atoms in the STEM images of the as-fabricated nanowires and DFT calculations. DFT simulations further shows a weaker interaction between the Se (W) and the Mo (S) atoms, leading to a reduced density of states near the Fermi level of the alloyed nanowires. Therefore, the electronic structure of the alloyed nanowires is sensitive to the alloyed concentration, giving rise to the opportunity of regulating their transport properties by manipulating the chemical composition of these alloyed nanowires.

The capping S (Se) atoms can be frequently removed by the electron beam and re-cap owing to the low diffusion barrier (a self-healing process). By directly mapping the chemical composition variation during the fabrication of $\mathrm{MoS}_{1-\mathrm{x}} \mathrm{Se}_{\mathrm{x}}$ alloyed nanowires (Figure 2), we further show that the Se concentration in MoS nanowire can be controlled by the acceleration voltage of the electron beam, demonstrating the possibility in manipulating the chemical composition of these alloyed nanowires during the fabrication process $[8,9]$.

References: 
[1] Lin. J.H. et al., Nature Nanotechnology, 2014. 9, 432

[2] Kibsgaard, J., et al., Nano Letters, 2008. 8, 3928

[3] Venkataraman, L., et al., Physical Review Letters, 1999, 83, 5334

[4] Meden, A., et al., Nanotechnology, 2005, 16, 1578

[5] Venkataraman, L., et al., Physical Review Letters, 2006, 96, 76607

[6] Liu, X., et al., Nature Communication, 2013, 4, 1776

[7] Lehtinen, O., et al., ACS Nano, 2015, 9, 3274

[8] Lin. J.H., et al., ACS Nano, 2016, DOI: 10.1021/acsnano.5b07888

[9] JL from AIST acknowledge support from the JST Research Acceleration Programme. This research was also supported in part by U.S. DOE grant DE-FG02-09ER46554 (JL, STP), by the U.S. Department of Energy, Office of Science, Basic Energy Science, Materials Sciences and Engineering Division (WZ), and through a user project at ORNL's Center for Nanophase Materials Sciences (CNMS), which is a DOE Office of Science User Facility. This research used resources of the National Energy Research Scientific Computing Center, which is supported by the Office of Science of the US Department of Energy under Contract No.DE-AC02-05CH11231.

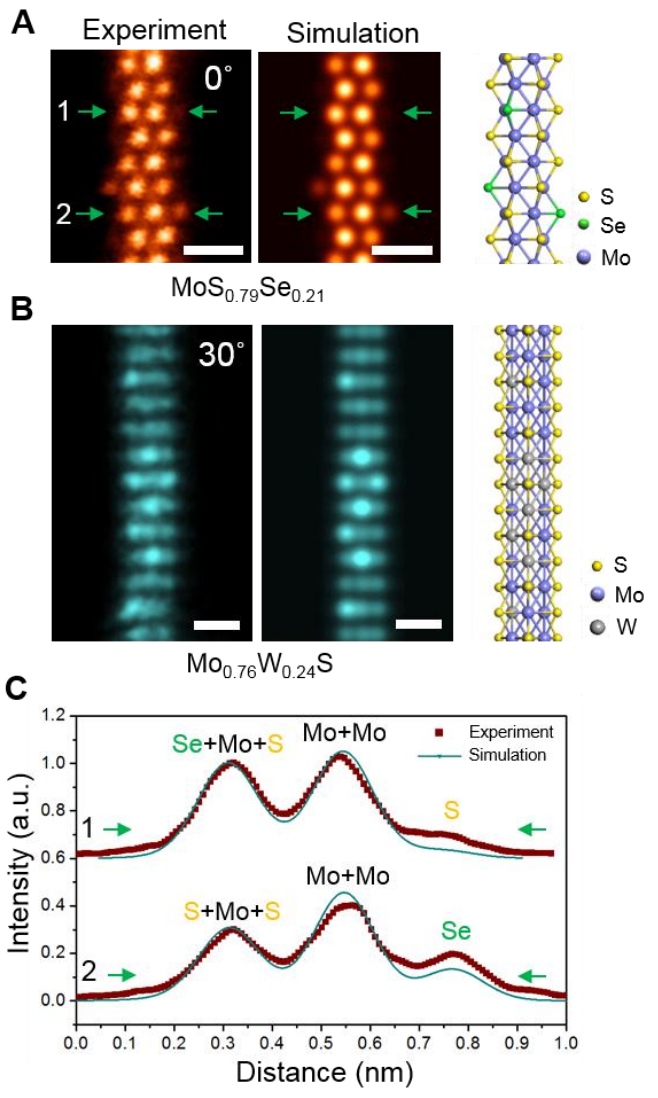

Figure 1: (A, B) Z-contrast STEM images of an alloyed $\mathrm{MoS}_{0.79} \mathrm{Se}_{0.21}$ at $0^{\circ}$ and $\mathrm{Mo}_{0.76} \mathrm{~W}_{0.24} \mathrm{~S}$ nanowire at $30^{\circ}(\mathrm{B})$ rotation angle, respectively. The atomic composition can be mapped out at each atomic column by their distinctive image intensity. (C) Intensity line profiles of the regions highlighted by the arrows in (A). Scale bars: $0.5 \mathrm{~nm}$. Figures reproduced from Ref. [8].
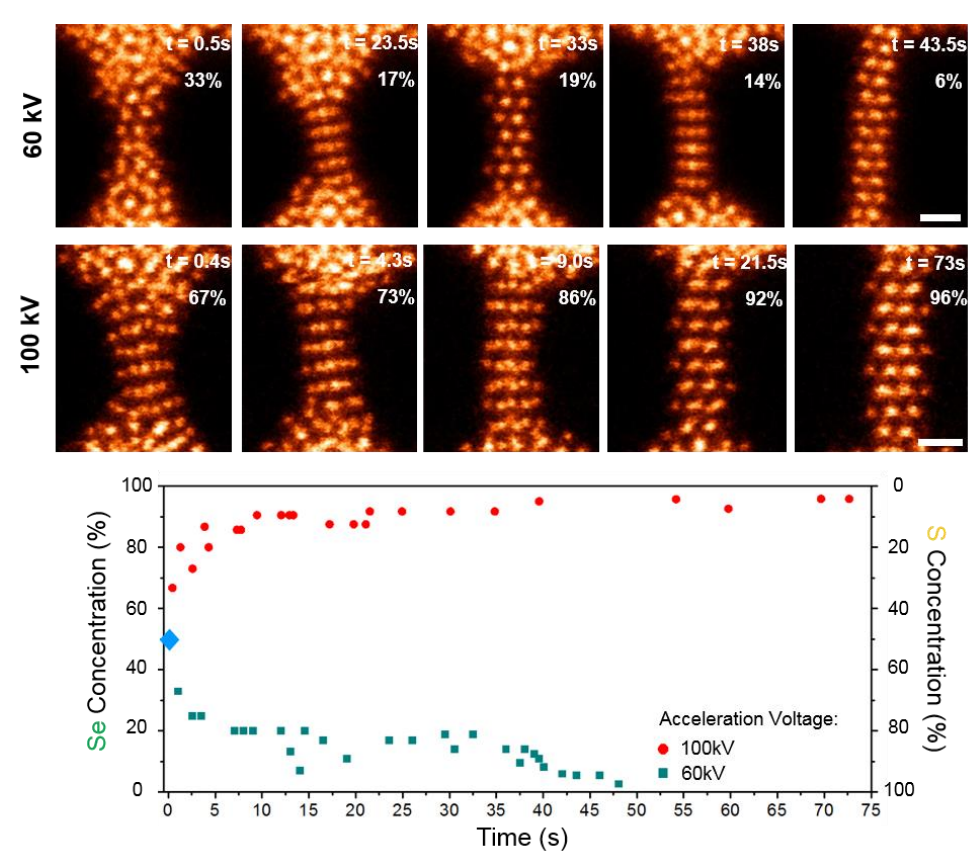

Figure 2: Two rows on the top: snapshots of the sequential Z-contrast STEM imaging of the dynamical formation process of two different alloyed $\mathrm{MoS}_{\mathrm{x}} \mathrm{Se}_{1-\mathrm{x}}$ nanowires sculpted from a $\mathrm{MoS}_{2} / \mathrm{MoSe}_{2}$ bilayer vertically stacked heterostructure under $60 \mathrm{kV}$ (first row) and $100 \mathrm{kV}$ (second row), respectively. The overall composition of the starting bilayer material is MoSSe, as indicated by the blue diamond symbol. The graph below summarized the evolution of the Se alloying concentration in the two nanowires as a function of time. The chemical composition of the nanowire is estimated frame-by-frame by intensity quantification analysis. Scale bars: $0.5 \mathrm{~nm}$. [8] 